\title{
A CASE OF OCULAR MYIASIS
}

BY

\author{
E. E. CASS
}

GIBRALTAR

OCular myiasis is rarely seen in Western Europe. It usually occurs in hot countries, where there are many flies, and the living and sanitary conditions are bad, and the people dirty and illnourished.

The most common fly which causes myiasis belongs to the muscoid group, amongst which the common house fly is the most usual cause of the trouble; all of this group lay eggs, usually choosing filthy situations, such as manure heaps, decomposing flesh, festering wounds; and sometimes the lids of people who are in a debilitated filthy state, with discharging eyes. If these flies do lay their eggs in the conjunctival sac, the majority lay them on the surface of the conjunctiva, but some are more dangerous, as their maggots burrow below the conjunctiva and penetrate the globe.

The oestridae possess ovipositors, and can lay their eggs beneath the conjunctiva itself, and one of them, the hypoderma bovis, can penetrate the globe. Luckily such cases of intra-ocular myiasis are very rare.

The sarcophagidae and anthomydae can also infect the conjunctiva of man.

The severity of the condition depends on the type of insect which has laid its egg in the eye, the condition of the patient, and, naturally, the longer the patient is left without treatment, the more severe is the reaction in the eye.

In the milder cases there is usually the history of a fly having entered the eye and being removed, but the discomfort does not diminish; there is increasing pain, lacrimation, and swelling of the lids and conjunctiva, and on examination the maggots are seen crawling in the eye. With removal of all the maggots the condition subsides. In the filthy ill-nourished patients who do not receive treatment, with numerous maggots, the larvae may hatch out and the whole eye be destroyed by the flies, and the orbit itself invaded, with consequent meningitis and death.

In cases of intra-ocular myiasis, intense pain and other symptoms of iritis are present. The parasite can be removed if it is in the anterior chamber, with some conservation of vision, but if it in the retina, enucleation has to be performed. 32 such cases have been described, of which 21 occurred in the anterior chamber. In 
the past 18 months 3 cases have been described in the Archivos de la Sociedad Oftalmologica Hispano-Americana; in two, larval conjunctivitis occurred, and in one of these, the patient had preexisting trachoma. Removal of the parasites resulted in relief of the symptoms. The third case was that of an intra-ocular myiasis caused by hypoderma bovis. Removal from the angle of the A.C. of a mass, containing the parasites, resulted in relief of. pain and a rapid subsidence of ocular signs and gradual return of normal vision.

The only case which has been recorded in Gibraltar is as follows: a healthy male Spaniard of 35 years was passing near a cement mixer, when he complained that some of the dust blew into his eye. A friend washed out his eye with water from a nearby tap. This occurred at 5 p.m., and the eye was immediately painful. By $10 \mathrm{p.m}$. he was suffering from intense pain, and could not sleep. His wife looked in his eye, and removed two small white bodies from it, but the pain still persisted. On the following morning he came to the Colonial Hospital, Gibraltar, sent up as a "cement burn" and immediately had his eye washed out. The nurse appeared in great agitation, saying that the man had "some white things walking in his eye." On examination he had swelling of the lids, with blepharo-spasm, pain and photophobia. The conjunctiva was oedematous and very inflamed. One small maggot, about $2 \mathrm{~mm}$. long, was seen walking across the cornea, and was removed with a rod. It was pear shaped and, with the loupe, a depression was seen on the under surface of the head together with tiny suckers. The head of another maggot was seen emerging from a burrow in the conjunctiva. Cocaine 5 per cent. was inserted, and seven maggots were removed with a needle, and sent to the laboratory for identification.

The eye was irrigated with hyd. perchlor. $(1: 10,000)$, and the patient was left to wait for an hour, and then examined again. Another maggot was then removed: this had burrowed into the conjunctiva and had to be levered out.

The patient again was examined after another hour, but no further maggots were found, so the eye was irrigated, oil inserted, a flap applied, and he was allowed to return home. The next day the eye was still injected in the lower fornix, and was still slightly painful, but no more larvae were found. $\mathrm{He}$ was ordered irrigations and oil. Two days later there was no pain, and only slight injection below.

In this case 11 maggots in all were found. The greatest number reported in larval conjunctivitis has been $7 \dot{7}$.

The man was seeking compensation, and insisted that no fly had been in his eye, but that the maggots must have been in the cement mixture, or the tap-water. The larvae were identified as those of Phlebotonus (sand-fly) and both cement and the tap water were examined for larvae with negative result.

It is probable that the small fly entered his eye with the dust, and was not seen by the friend who washed out the eye.

\section{LITERATURE}

DUKE-ELDER, W. S.-Textbook of Ophthalmology, 2, 1675-8.

INIGo, L. (Santander) (1948). - Oftalmoeuliasis interna, Arch. de la Soc. Oftal. Hisp.-Amer., 8.

Guinea, A. Castresana y (1947).-Miasis Ocular, Ibid., 7.

VAldecasas, PEDro.-Larviasis Ocular, Ibid., 7. 